\title{
Aumento da concentração de vapor de água atmosférico na Groenlândia
}

\author{
Increase of concentration of water vapor atmospheric in the Greenland
}

\author{
Henderson Silva Wanderley ${ }^{1}$ e Flávio Barbosa Justino ${ }^{2}$ \\ ${ }^{1}$ Departamento de Ciências Ambientais, Universidade Federal Rural do Rio de Janeiro, (DCA/IF/UFRRJ), Rodovia \\ BR 465, Km 07 Seropédica - RJ, CEP 23890-000, Seropédica, RJ. E-mail: henderson@ufrri.br \\ ${ }^{2}$ Departamento de Engenharia Agrícola, Universidade Federal de Viçosa, (DEA/UFV), Av. P.H. Rolfs, s/n, Campus \\ Universitário, CEP 36570-900, Viçosa, MG. E-mail:fjustino@ufv.br
}

\begin{abstract}
Resumo
O vapor de água desempenha um papel importante na condução de circulações atmosféricas por meio da liberação de calor latente e na determinação de saldo de radiação do planeta. O feedback do vapor de água é o mais importantes em nosso sistema climático, com capacidade de dobra o aquecimento direto do aumento de gases de efeito estufa, sendo um dos mais importantes constituintes atmosféricos necessários para o estudo das mudanças climáticas, principalmente para as região Ártica. Deste modo, o objetivo deste estudo consiste em analisar a existência de tendência na concentração de vapor de água na Groenlândia. Foram utilizados dados mensais de radiossonda, entre o período de 1980-2010, para o nível atmosférico de 700 e 500 hPa, na região da Groenlândia. Para este fim, os dados foram submetidos à aplicação do teste estatístico não paramétrico de Mann-Kendall, a um nível de significância estatística de 5\%. Os resultados mostram que todos os meses apresentam tendência para o aumento do vapor de água na atmosfera, com tendência estatisticamente significativa para os meses de janeiro, março, abril e dezembro nos níveis analisados.
\end{abstract}

Palavras-chave: Tendência. Feedback positivo. Mudanças climáticas

\begin{abstract}
Water vapor plays an important role in driving atmospheric circulations through latent heat release and in determining the earth's radiation budget. The water vapor feedback is the most important in our climate system, with the capacity to about double the direct warming from greenhouse gas increases, one of the most important atmospheric constituents needed for studies of climate change mainly for the Arctic region. Thus, the objective this study was to analyze trend presence in the of water vapor concentration in Greenland. We use month's information from radiosonde to period of 1980-2010 in atmospheric level of 700 and $500 \mathrm{hPa}$, in Greenland region. For this goal, the data were subjected to statistical test nonparametric Mann-Kendall to statistical significance of $5 \%$. The results show that every month has trend to increase the water vapor in the atmosphere with statistically significant trend for the months of january, march, april and December levels analyzed.
\end{abstract}

Keywords: Trend. Positive feedback. Climate change 


\section{INTRODUÇÃO}

O vapor de água atmosférico é um constituinte importante por interferir na dinâmica e na química da atmosfera, agindo na distribuição da temperatura, participando ativamente dos processos de absorção e emissão de calor sensível na atmosfera e por atuar na transferência de calor latente entre as regiões. Além disso, destaca-se sua contribuição no efeito estufa, sendo este seu principal causador. Além disso, é o único constituinte atmosférico que muda de estado físico, sendo responsável pela formação e evolução das nuvens e por seus fenômenos atmosféricos importantes como a precipitação, sendo ela na forma líquida ou sólida.

O vapor de água é responsável pela absorção de $50 \%$ da radiação solar atmosférica e pela maior parte da radiação infravermelha emitida pela superfície da terra, sendo o componente atmosférico mais eficaz para o efeito estufa (COHEUR et al., 2003). Deste modo, eventuais distúrbios na concentração de vapor de água atmosférico, como o seu aumento, pode contribuir na absorção da radiação solar atmosférica modificando o seu balanço e as principais reações físico-químicas existentes na atmosfera. Outra característica importante do vapor de água atmosférico é que o mesmo apresenta respostas diretas ao aumento da temperatura, aumentando sua concentração na atmosfera, e por consequência sua contribuição para aquecê-la, em função da intensificação do efeito estufa (RANDALL et al., 2007).

A absorção da radiação de onda curta pelo vapor de água na atmosfera é aproximadamente de 5 a 10 vezes menor do que os calculados para a radiação de onda longa. No entanto, para as latitudes polares esse valor é maior, assim, a sensibilidade da radiação de ondas curtas é maior nos níveis mais baixos, onde a concentração de vapor é maior (SODEN et al., 2008). Essa maior absorção é decorrente do reflexo da radiação incidente sobre a superfície coberta de neve e/ou gelo das regiões polares, o que aumenta a absorção da radiação refletindo de volta para a atmosfera.

Já a radiação de onda longa tem sua maior absorção nos níveis mais alto da atmosfera. Como o aprisionamento dessa radiação está associado à intensificação do efeito estufa, a aumento de vapor de água nestes níveis apresenta-se como agravante ao efeito estufa e seus distúrbios causados no clima, o que pode ser um dos indicativos do aumento da temperatura observada no Ártico (PARMENTIER et al., 2013). Outro agravante a presença de vapor de água na atmosfera se deve a presença de nuvens. As nuvens atuam para intensificar o feedback do vapor de água na atmosfera, aumentando sua absorção e o caminho óptico do espectro solar na atmosfera por meio da reflexão. A presença de nuvens serve para aumentar a absorção de radiação em relação às condições de céu sem nuvem e ao mesmo tempo diminuir a absorção nos níveis mais baixos.

A atuação do vapor de água na atmosfera vem sendo largamente discutidas, sobre tudo no que diz respeito da atuação do vapor de água como feedback positivo, ou seja, sua capacidade de amplificar a ação dos gases de efeito estufa. A ação do vapor de água como feedback positivo foi ratificada por estudos como o de Dessler et al. (2009), os quais encontraram mudanças no balanço de radiação decorrente da presença do vapor de água de 2,04 $\mathrm{Wm}^{-2} \mathrm{~K}^{-1}$, entre os anos de 2003 a 2008.

Este estudo mostra concordância como os de Forster e Collins (2004) e Minschwaner et al. (2006), que também ratificam a ação do vapor de água atmosférico como feedback positivo. Segundo Houghton et al. (2001), a forçante radiativa obtida pelo aumento da concentração de dióxido de carbono $\left(\mathrm{CO}_{2}\right)$ entre os anos de 1750 a 2000 , foi igual a $1,5 \mathrm{Wm}^{-2}$. Estes resultados demonstram a importância do vapor de água na atmosfera e ressalta sua atuação no sistema climático.

Para o hemisfério norte tem se observado um aumento do aquecimento com o aumento da latitude, sobre tudo para grande parte do oceano Ártico durante vários meses do ano. Esse aquecimento é atribuído à redução de neve na superfície e da espessura do gelo dos oceanos, modificação no albedo, mudança da cobertura de nuvens, mudanças na circulação atmosférica e oceânica além do aumento da concentração vapor de água (GILLETT et al., 2008; MANABE et al., 2011).

As mudanças observadas no Ártico fazem com que grande parte da superfície durante o inverno aqueça, e seja observado elevado índice de evaporação, que por sua vez, aumenta a concentração de vapor de água atmosférica, intensificando o aquecimento da mesma. No entanto, o número de pesquisas que se detém a estudar a variabilidade do vapor de água nas regiões polares é escassa devido sua ampla importância para o sistema climático. Deste modo, o objetivo deste estudo consiste em analisar a existência de tendência na concentração de vapor de água na atmosfera de Groenlândia.

\section{MATERIAL E MÉTODOS}

Para o desenvolvimento deste estudo foram utilizadas informações do perfil vertical do vapor de água troposférico, avaliado por meio da razão de mistura (r). Foram utilizados dados mensais de radiossonda, entre o período de 1980-2010, para o nível atmosférico de 700 e 500 hPa. As radiossondagens foram realizadas na estação 04360 Tasiilaq, localizada no sudeste da Groelândia, Figura 1. As medições estão disponíveis no banco de dados de Universidade de Wyoming (http://weather.uwyo.edu/ upperair/sounding.html).

Para a identificação de tendência na série temporal de razão de mistura foi usado o teste estatístico não paramétrico de Mann-Kendall (MANN, 1945; KENDALL, 1975), também conhecido como teste "S". O teste considera a hipótese de estabilidade de uma série temporal, onde a sucessão de valores ocorre de forma independente e a 
distribuição de probabilidade deve permanecer sempre a mesma (série aleatória simples). O teste considerando a série temporal Yi de $\mathrm{n}$ termos $(1 \leq \mathrm{i} \leq \mathrm{n})$, onde a estatística desse teste é dada pela Equação (1).

$$
S=\sum_{i=1}^{n-1} \sum_{j=i+1}^{n} \operatorname{sinal}\left(Y_{j}-Y_{i}\right)
$$

em que:

$$
\operatorname{sinal}\left(Y_{j}-Y_{i}\right)=\left\{\begin{array}{ll}
+1 & \operatorname{se}\left(Y_{j}-Y_{i}\right)>0 \\
0 & \operatorname{se}\left(Y_{j}-Y_{i}\right)=0 \\
-1 & \operatorname{se}\left(Y_{j}-Y_{i}\right)<0
\end{array}\right\} \text { Para séries }
$$

com grande número de termos (n), a estatística "S" apresenta uma distribuição normal com média zero e variância definida pela Equação (2).

$$
\operatorname{Var}(S)=\frac{n(n-1)(2 n+5)}{18}
$$

O teste tem como hipótese nula $\left(\mathrm{H}_{\mathrm{o}}\right)$ a ausência de tendência na população. Testando a significância estatística de $\mathrm{S}$ para a hipótese alternativa $\left(\mathrm{H}_{1}\right)$, a qual expressa à existência de tendência, usa-se um teste bilateral a $\mathrm{H}_{1}$, pelo qual a hipótese alternativa pode ser rejeitada para grandes valores da estatística Z, Equação (3).

$$
z= \begin{cases}\frac{(S-1)}{\sqrt{\operatorname{Var}(S)}} & \text { se } S>0 \\ 0 & \text { se } S=0 \\ \frac{(S+1)}{\sqrt{\operatorname{Var}(S)}} & \text { se } S<0\end{cases}
$$

Com base na análise da estatística $\mathrm{Z}$, é realizada a decisão de aceitar ou rejeitar a hipótese nula $\left(\mathrm{H}_{\mathrm{o}}\right)$ de ausência de tendência, confirmar a hipótese de estabilidade dos dados ou rejeitá-la a favor da hipótese alternativa $\left(\mathrm{H}_{1}\right)$, admitindo a existência de tendência na série dados.

O sinal da estatística $Z$ indica se a tendência é crescente $(Z>0)$ ou decrescente $(Z<0)$. Em um teste bilateral para tendência, $H_{1}$ deve ser aceita se $|Z| \geq Z_{\alpha / 2^{\prime}}$ em que o valor de $Z_{\alpha / 2}$ é obtido da tabela na distribuição normal padronizada. Para esse teste foi adotado nível de significância de $\alpha=0,05$, que apresenta intervalo para $Z$ tabelado de $\pm 1,96$.

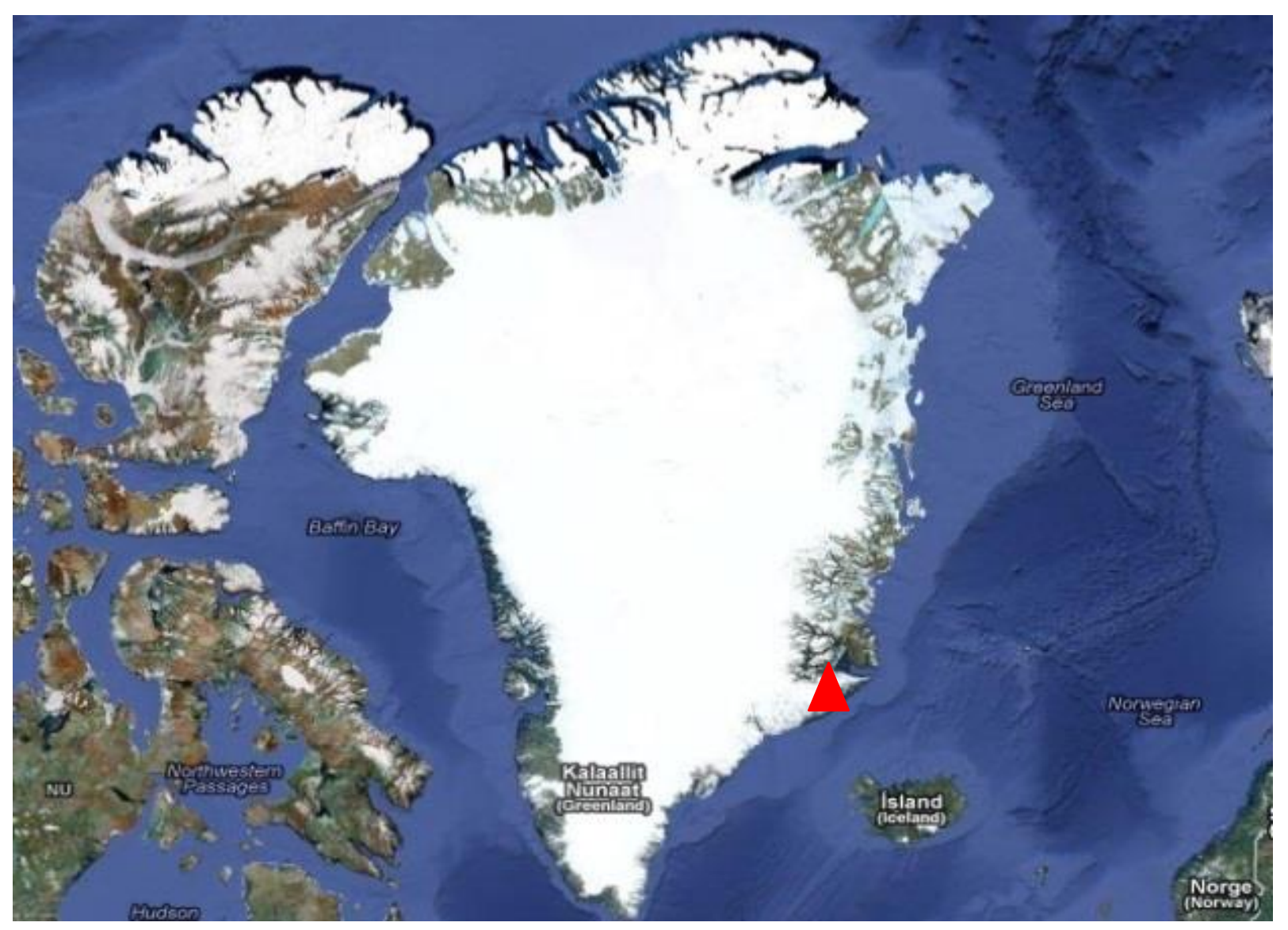

Figura 1 - Identificação do local de medição do vapor de água. 


\section{Resultados e discussões}

As medições de vapor de água realizadas na atmosfera da Groenlândia demostraram aumento na concentração de vapor de água atmosférico em todos os meses analisados. Para a Groenlândia, o aumento na concentração vapor de água mensal foi ratificado pela aplicação do teste de Mann-Kendall, que demonstrou tendência positiva para todos os meses. Parte do aumento mensal do vapor de água apresentou significância estatística, sobretudo para o período de inverno no hemisfério norte, Tabela 1.

O acréscimo do vapor de água na atmosfera da Groenlândia, para os dois níveis em análise, demonstrou padrão semelhante de tendência, o que também pode ser observado para os meses que apresentaram significância estatística. Para o nível de $700 \mathrm{hPa}$, nos meses de dezembro, janeiro, março, abril e agosto foi identificado tendência estatisticamente significativa. Para o nível de $500 \mathrm{hPa}$, dos meses que apresentaram tendência significativa em $700 \mathrm{hPa}$, apenas no mês de agosto não foi identificada tendência com significância. No entanto, foi observada tendência estatisticamente significativa no mês de setembro, no nível de $500 \mathrm{hPa}$.

As tendências obtidas para a concentração de vapor de água na Groenlândia é reflexo das mudanças que estão sendo observadas no Ártico. A região em análise na Groenlândia é uma das áreas onde se observou as maiores mudanças, principalmente com relação ao aumento da temperatura. Não obstante disto, para essa região se projeta um aumento do número de dias quentes e de precipitação na forma líquida com redução na precipitação em forma de neve (STENDEL at al., 2008). O aumento da temperatura do Ártico proporciona uma maior retenção de vapor de água pela atmosfera, como foi observado nos resultados apresentados na Tabela 1.

O maior número de tendências com significância estatística foi observada para a estação de inverno no hemisfério norte. Também neste período, para a atmosfera do Ártico, tem se observado um aumento da temperatura acima de $700 \mathrm{hPa}$, ainda que esse seja mais fraco do que o observado perto da superfície (SCREEN e SIMMONDS, 2010). Esse aquecimento justifica parte das tendências estatisticamente significativas observadas neste período já que a atmosfera tem uma maior capacidade de reter vapor de água.

A maior parte do aquecimento de inverno está associada a alterações na cobertura de gelo do mar, embora esse declínio cause pequenos distúrbios em relação ao albedo durante esta temporada. Essa interação é, sem dúvida reciproca, pois a temperatura maior do oceano irá aumentar ainda mais a perda de gelo marinho. Deste modo, o excesso de calor armazenado na parte superficial dos oceanos é subsequentemente liberado para a atmosfera durante o inverno. Isso faz com que a cobertura de gelo no mar durante o inverno se reduza, facilitado uma maior transferência de calor e umidade para a atmosfera, como visto na Tabela 1 para o vapor de água na atmosfera. A presença dessa amplificação contribui para o aumento da transferência de calor do oceano para a atmosfera, resultando em perda de gelo no inverno e aumento do vapor para a atmosfera como observado em função das tendências obtidas.

Estes fatos indicam que o aumento de umidade no Ártico é resultante de interações locais e do aumento do transporte de umidade para o Ártico. Mudanças na cobertura de nuvens, também têm sido propostas como causa das mudanças observadas no Ártico (GRAVERSEN et al., 2008; SCREEN e SIMMONDS, 2010). No Ártico, as nuvens atuam amplificando o papel de feedback do vapor de água em relação a absorção do espectro solar, onde ao mesmo tempo, diminui a absorção nos níveis mais baixos da atmosfera (SODEN at el., 2008). No Ártico, exceto, por um curto período de verão, nuvens baixas persistentes induzem aquecimento da superfície (INTRIERI et al., 2002).

Segundo Graversen (2006) o derretimento da neve e o feedback do gelo não pode ser a principal causa do aquecimento no ar durante a maior parte do ano no Ártico. Isso se deve ao fato de que esses feedbacks devem afetar principalmente a temperatura na parte mais baixa da atmosfera, resultando em um padrão de aquecimento que só observa-se na primavera, com aumento de umidade. No entanto, a analise realizada no vapor de água mostrou uma maior mudança nos meses de inverno.

O aquecimento excessivo do Ártico se deu também devido ao aumento no transporte de calor e umidade para a atmosférica polar. Estes resultados são apoiados por estudos observacionais, o qual sugere que as mudanças do transporte de calor contribuíram para o recente aquecimento da superfície do Ártico (GRAVERSEN et al., 2008). A ligação entre o aquecimento do Ártico e as mudanças da circulação atmosférica tem sido investigada e correlacionada com vários índices de circulação do hemisfério norte, como a Oscilação do Ártico (OA) e a Oscilação do Atlântico Norte (NAO, sigla em inglês).

A Oscilação do Ártico representa a variabilidade da circulação atmosférica de todo o hemisfério norte, sendo

Tabela 1: Tendência mensal do vapor de água na atmosfera da Groenlândia

\begin{tabular}{ccccccccccccc}
\hline Níveis & Jan & Fev & Mar & Abr & Mai & Jun & Jul & Ago & Set & Out & Nov & Dez \\
\hline 700 & $2,46^{*}$ & 1,68 & $3,28^{*}$ & $1,98^{*}$ & 0,81 & 1,71 & 1,34 & $2,37^{*}$ & 1,95 & 0,98 & 1,22 & $2,26^{*}$ \\
500 & $2,40^{*}$ & 1,58 & $2,81^{*}$ & $3,00^{*}$ & 1,22 & 0,52 & 1,46 & 1,51 & $2,14^{*}$ & 0,95 & 1,37 & $2,61^{*}$ \\
\hline
\end{tabular}


responsável pela transferência de massa atmosférica entre regiões de alta pressão subtropical e baixas polares. Devido à geometria complexa dos continentes do norte, a Oscilação do Ártico é especialmente forte sobre o Atlântico norte e menos evidente em outras regiões. Em sua fase positiva, a Oscilação do Ártico aumenta a retenção de ar frio no Ártico, aumento o fluxo norte do jato de oeste, causando baixa temperatura e alta precipitação em forma de neve.

A mudança na circulação atmosférica associada à redução do gelo marinho mostra resultado em episódios de bloqueio atmosféricos mais frequentes, que levam a mudança na circulação em grandes partes dos continentes do norte. Em regiões como o nordeste do Canadá e Groenlândia, os ventos de oeste favorecem incursões mais frequentes de massas de ar quentes do Atlântico norte, a qual aumenta a quantidade de vapor de água na região (JACKSON et al., 2010). Esses indicativos fortalecem a influencia da circulação atmosférica no aumento de vapor de água na Groenlândia.

Outra condição que pode ter contribuído para o aumento do vapor de água foi à mudança de fase na Oscilação do Atlântico Norte (NAO) ocorrida em 1997. A fase positiva da NAO está associada com ventos de oeste mais forte do que a média da superfície, com o fluxo meridional anômalo sobre o leste dos Estados Unidos e fluxo de norte anômalo no Ártico. Quando se compara seus regimes, observam-se poucas ocorrências de sua fase positivas durante a década de 1960, e muito poucas ocorrências das fases negativas durante a década de 1990.

Com a mudança em sua fase, o fluxo pode ter enfraquecido e possivelmente mudados, contribuído para o aumento de vapor de água no Ártico, uma vez que, o Modo Anular do Hemisfério Norte em sua fase negativa possibilita a entrada de ar mais quente, oriundos dos sistemas de alta pressão de latitudes médias, em direção ao norte. Como a NAO é o modo dominante de variabilidade regional do hemisfério norte, sua mudança contribui para modificar o padrão local observado durante suas fases aumentando o fluxo de umidade para o Ártico (HURRELL e DESER, 2009).

Quando a diferença de pressão no inverno é grande, entre Portugal e Islândia, a frequência de tempestades intensas é maior na faixa nordeste no Atlântico norte. Sobre o norte da Europa o clima é mais quente e úmido, e são observadas condições frias e secas no norte do Canadá, condição mais branda de umidade ao longo da costa leste dos Estados Unidos e aumento da advecção de calor e umidade para o Ártico (HURRELL e DESER, 2009).

\section{Conclusões}

Os resultados encontrados com este estudo mostraram que em todos os meses analisados foi ratificada a presença de tendência positiva, a qual ratifica o aumento do vapor de água na atmosfera na Groenlândia. A presença de tendência significativa foi observada, em sua grande maioria, para os meses de inverno do hemisfério norte.

O padrão de aumento na concentração de vapor de água na atmosfera foi observado para os dois níveis em análise. Para o nível de 700 hPa, foi observado tendência estatisticamente significativa para os meses de dezembro, janeiro, março, abril e agosto. Padrão análogo foi observado para o nível de $500 \mathrm{hPa}$, no entanto com a exclusão do mês de agosto e a inclusão do mês de setembro.

\section{Referências}

COHEUR, P. F. C.; CLERBAUX, C.; CARLEER, M.; FALLY, S.; HURTMANS, D.; COLIN, R.; HERMANS, C.; , VANDAELE, A. C.; BARRET, B.; MAZIÈRE, M.; BACKER, H. Retrieval of atmospheric water vapor columns from FT visible solar absorption spectra and evaluation of spectroscopic databases. Journal of Quantitative Spectroscopy \& Radiative Transfer, vol. 82, p.133-150, 2003.

DESSLER, A. E.; WONG, S. Estimates of the Water Vapor Climate Feedback during El Niño-Southern Oscillation. Journal of Climate, vol. 22, p. 6404-6412, 2009.

FORSTER, P. M. F.; COLLINS, M. Quantifying the water vapor feedback associated with post-Pinatubo global cooling. Climate Dynamics, vol. 23, p. 207-214. 2004.

GRAVERSEN, R. G. Do changes in the midlatitude circulation have any impact on the Arctic surface air temperature trend. Journal Climate, vol. 19, p. 54225438, 2006.

GRAVERSEN, R. G.; MAURITSEN, T.; TJERNSTRÖM, M.; KÄLLÉN, E.; SVENSSON, G. Vertical structure of recent Arctic warming. Nature, vol 541, p. 53-57, 2008.

GILlETT, N. P.; STONE, D. A.; STOTT, P. A. NOZAWA, T.; KARPECHKO, A. Y.; HEGER, G. C. WEHNER, M. F.; JONER, P. D. Attribution of polar warming to human influence. Nature Geoscience, vol 1, p. 750-754. 2008.

HOUGHTON, J. T., Y. DING, D. J. GRIGGS, M. NOGUER, P. J. VAN DER LINDEN, X. DAI, K. MASKELL, AND C. A. JOHNSON, E. Climate Change 2001: The Scientific Basis. Cambridge University Press, 881 pp, 2001.

HURRELL, J. W.; DESER, C. North Atlantic climate variability: The role of the North Atlantic Oscillation. Journal of Marine Systems, vol. 78, p. 28-41, 2009.

INTRIERI, J. M., C. W. FAIRALL, M. D. SHUPE, P. O. G. PERSSON, E. L. ANDREAS, P. S. GUEST, AND R. E. MORITZ. An annual cycle of Arctic surface cloud 
forcing at SHEBA, Journal of Geophysical Research, vol. 107, p. 8039. 2002.

JACKSON J, CARMACK E, MCLAUGHLIN F, ALLEN S. Ingram R (2010) Identification, characterization, and change of the near surface temperature maximum in the Canada Basin,1993-2008. Journal of Geophysical Research, vol. 10, p. 1029-2009. 2010.

KENDALL, M. G. Rank correlation methods. 4. ed. London: Charles Griffin, 1975. 196p.

MANN, H. B. Non-parametric test against trend. Econometrika, v.13, p.245-259, 1945.

MANABE, S.; PLOSHAY, J.; LAU, N, C. Seasonal variation of surface temperature change during the last several decades. Journal of Climate, vol. 24, p 3817-3821. 2011.

MINSCHWANER, K.; DESSLER. A. E.; AENGPHOKHAI, P. S. Multimodel Analysis of the Water Vapor Feedback in the Tropical Upper Troposphere. Journal of Climate, vol. 19, p. 5455-5464, 2006.

PARMENTIER, F. J. W.; CHRISTENSEN, T. R.; SORENSEN, L. L.; RYSGAARD, S.; MCGUIRE, D.; MILLER, P. A.; WALKER, D. A. The impact of lower sea-ice extent on Arctic greenhouse-gas Exchange. Nature Climate Change, vol. 3, p. 195-202. 2013.

RANDALL, D. A. Climate models and their evaluation, in Climate Change 2007: The Physical Science Basis. Ontributions of Working Group I to the Fourth Assessment Report of the Intergovernmental Panel on Climate Change, edited by S. Solomon et al., p. 591- 662, 2007.

SCREEN, J. A.; SIMMONDS, I. The central role of diminishing sea ice in recent Arctic temperature amplification. Nature, vol. 464, p. 1334-1337, 2010.

STENDEL, M.; CHRISTENSEN, J. H.; PETERSEN, D. Arctic Climate and Climate Change with a Focus on Greenland. Review Article Advances in Ecological Research, vol. 40, p. 13-43. 2008.

SODEN, B. J.; HELD, I. M.; COLMAN, R. Quantifying Climate Feedbacks Using Radiative Kernels. Journal of Climate, vol. 21, p. 3504-3520, 2008. 